\title{
Eco-friendly and efficient synthesis of bis(indolyl)methanes under microwave irradiation
}

\author{
Magdy Zahran," Yasmin Abdin, and Hanan Salama \\ Organic Chemistry Laboratory, Department of Chemistry, Faculty of Science, \\ Menoufiya University, Egypt \\ E-mail: magdyzahran@gmail.com
}

\begin{abstract}
Treatment of 2-arylindole derivatives with structurally diverse aldehydes in the presence of glacial acetic acid as an efficient, mild, and inexpensive catalyst under microwave irradiation condition compared with the conventional method afforded excellent yields of biologically important bis(indolyl)methane and tetraindolyl(terephthalyl)dimethane derivatives.
\end{abstract}

Keywords: Microwave irradiation, bis(indolyl)methanes, 2-arylindoles, aldehydes

\section{Introduction}

The indole moiety is featured in a variety of pharmacologically and biologically active compounds. ${ }^{1}$ Among various indole derivatives, di(1- $H$-indolyl-3-yl)methanes (DIM) and 1,4bis[di(1H-indol-3-yl)methyl]benzenes display diverse pharmacological activities and are useful in the treatment of fibromyalgia, chronic fatigue and irritable bowel syndrome. ${ }^{2}$ These compounds also inhibit the proliferation of both estrogen dependent and independent cultured breast tumor cells. ${ }^{3,4}$ Thus, the development of high-throughput methods for the synthesis of bis(indolyl)methanes remains a topic of paramount importance in view of their versatile biological and pharmacological activities. Numerous methods describing the synthesis of bis(indolyl)methanes were reported in the literature employing protic acids ${ }^{5}$ and Lewis acids. ${ }^{6,7}$ However, there are still some drawbacks in these catalytic systems including the requirement of large, ${ }^{8,9}$ or stoichiometric amount of catalysts, ${ }^{10,11}$ long reaction times, ${ }^{8,9}$ low yields of products ${ }^{11}$

and drastic condition for catalyst preparation. ${ }^{12}$ Recently, metal triflate in ionic liquid, ${ }^{13} \mathrm{Fe}(\mathrm{III})$ salts in ionic liquids ${ }^{14}$ and ionic liquids ${ }^{15}$ were reported to be efficient for this transformation. Although ionic liquids are reusable they are very expensive.

During the past two decades many publications have described the successful combination of microwave irradiation as a nonclassical energy source with alternative reaction media. Microwave irradiation is well known to promote the synthesis of a variety of compounds, ${ }^{16,17}$ 
where chemical reactions are accelerated because of selective absorption of microwaves by polar molecules.

Recently, the coupling of microwave irradiation with polar organic molecules under solventfree conditions has received notable attention. ${ }^{17}$ A literature survey reveals examples of specific reactions, which do not occur under conventional heating, but could be possible by microwave irradiation. $^{18}$

In continuation of our interest on indole derivatives ${ }^{19}$ as well as the utility of microwave synthesis under solvent-free conditions, ${ }^{20}$ we focus in this article on an efficient and facile microwave irradiation synthesis of pharmacologically interesting di(1H-indol-3-yl)methane and 1,4-bis[di(1H-indol-3-yl)methyl]benzene derivatives.

\section{Results and Discussion}

In the present article, a facile route using glacial acetic acid as a mild and highly efficient catalyst for a comparative synthesis of di( $1 H$-indol-3-yl)methanes 3 by conventional heating and under microwave irradiation condition were described (Scheme 1).
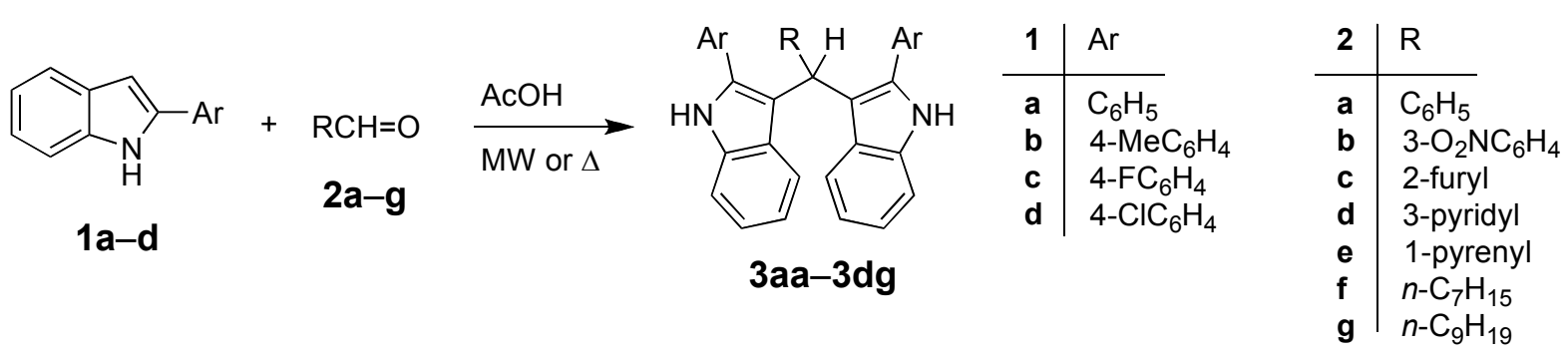

Scheme 1. Glacial acetic acid catalyzed synthesis of di(1H-indol-3-yl)methanes 3.

Attempts to synthesize some known di(1H-indol-3-yl)methane derivatives using catalysts such as $\mathrm{I}_{2},{ }^{21}$ silica sulfuric acid, ${ }^{22} \mathrm{HClO}_{4}-\mathrm{SiO}_{2}{ }^{23}$ under thermal conditions, revealed that the reactions took very long, required a huge amount of catalyst more than the reported, afforded low to moderate yields, and in some cases many by-products were formed.

In comparison with the reported methods, glacial acetic acid turned out to be an efficient medium in terms of handling, yields, and reaction times when carrying out the reactions under microwave irradiation. Thus, a mixture of 2-arylindole derivative $\mathbf{1 a}-\mathbf{d}$ and aldehyde $\mathbf{2 a}-\mathbf{g}$ (2:1 $\mathrm{mmol})$ in glacial acetic acid was subjected to microwave irradiation with successive $30 \mathrm{sec}$ periods to avoid overheating of the catalyst. The resulting di( $1 H$-indol-3-yl)methanes $\mathbf{3}$ were obtained in excellent yields especially with aromatic and heteroaromatic aldehydes, but in the case of aliphatic aldehydes the yields were moderate to good (Table 1). 
The work-up of these reactions is easy because some of the products either crystallized directly from the acetic acid, or upon pouring the reaction mixture onto water the solid product precipitated and was obtained by filtration and recrystallization.

By conventional heating condition in acetic acid di(1H-indol-3-yl)methanes 3 and 1,4bis[di(1H-indol-3-yl)methyl]benzene derivatives 5 were obtained in lower yields and required longer reaction times as compared with microwave irradiation (Table 1).

Table 1. Synthesis of di(1H-indol-3-yl)methanes $\mathbf{3}$ and tetraindolyl(terephthalyl)dimethanes 5 under thermal and microwave irradiation conditions

\begin{tabular}{|c|c|c|c|c|c|c|}
\hline \multirow{2}{*}{ Indole } & \multirow{2}{*}{ Aldehyde } & \multirow{2}{*}{ Product } & \multicolumn{2}{|c|}{ Thermal } & \multicolumn{2}{|c|}{ Microwave $(750 \mathrm{~W})$} \\
\hline & & & Time [min] & Yield $^{\mathrm{a}}[\%]$ & Time [min] & Yield $^{\mathrm{a}}[\%]$ \\
\hline $1 \mathbf{a}$ & $2 \mathbf{a}$ & $3 \mathbf{a a}^{c}$ & 30 & 84 & 3 & 96 \\
\hline $1 b$ & $2 \mathbf{a}$ & $3 \mathbf{b a}$ & 45 & 80 & 4 & 93 \\
\hline 1c & $\mathbf{2 a}$ & $3 \mathbf{c a}^{\mathrm{c}}$ & 15 & 63 & 1 & 91 \\
\hline 1d & $2 \mathbf{a}$ & 3da & 15 & 74 & 2 & 94 \\
\hline $\mathbf{1 a}$ & $2 b$ & $3 \mathbf{a b}$ & 20 & 70 & 3 & 96 \\
\hline $1 b$ & $2 \mathbf{b}$ & $3 \mathbf{b b}$ & 20 & 81 & 3 & 93 \\
\hline 1c & $2 \mathbf{b}$ & $3 \mathrm{cb}$ & 25 & 65 & 1 & 95 \\
\hline 1d & $2 b$ & $3 d b$ & 30 & 66 & 5 & 84 \\
\hline $1 \mathbf{a}$ & $2 c^{b}$ & $3 \mathbf{a c}^{\mathrm{c}}$ & 10 & 82 & 2 & 96 \\
\hline 1b & $2 c^{b}$ & $3 b c^{c}$ & 10 & 81 & 2 & 96 \\
\hline 1c & $2 c^{b}$ & $3 c c^{c}$ & 15 & 80 & 2 & 90 \\
\hline 1d & $2 c^{b}$ & 3dc & 20 & 75 & 2 & 91 \\
\hline $1 \mathbf{a}$ & $2 d$ & 3ad & 20 & 84 & 2 & 95 \\
\hline $1 b$ & $2 d$ & 3bd & 30 & 84 & 2 & 92 \\
\hline 1c & $2 d$ & $3 \mathrm{~cd}$ & 15 & 71 & 1 & 80 \\
\hline $1 d$ & $2 d$ & 3dd & 20 & 67 & 2 & 92 \\
\hline $1 \mathrm{a}$ & $2 \mathrm{e}$ & 3 ae & 258 & 37 & 5 & 80 \\
\hline $1 b$ & $2 e$ & 3be & 300 & 35 & 10 & 77 \\
\hline 1c & $2 \mathrm{e}$ & $3 c e$ & 720 & 53 & 5 & 79 \\
\hline 1d & $2 e$ & 3de & 750 & 64 & 7 & 80 \\
\hline $1 \mathrm{a}$ & $2 f$ & 3af & 480 & 43 & 10 & 63 \\
\hline $1 \mathrm{a}$ & $2 \mathrm{~g}$ & 3ag & 600 & 45 & 5 & 56 \\
\hline $1 d$ & $2 f$ & 3df & 480 & 44 & 5 & 62 \\
\hline $1 d$ & $2 \mathrm{~g}$ & $3 d g$ & 600 & 42 & 7 & 56 \\
\hline $1 \mathrm{a}$ & 4 & $5 \mathbf{a}$ & 120 & 92 & 10 & 95 \\
\hline $1 b$ & 4 & $5 b$ & 150 & 86 & 10 & 95 \\
\hline 1c & 4 & $5 c$ & 150 & 43 & 45 & 76 \\
\hline $1 \mathrm{~d}$ & 4 & $5 d$ & 160 & 40 & 60 & 66 \\
\hline
\end{tabular}

${ }^{\mathrm{a}}$ Yield of isolated products. ${ }^{\mathrm{b}}$ Irradiation power was $350 \mathrm{~W}$. ${ }^{\mathrm{c}} \mathrm{K}$ nown compounds. 
The reaction was further explored, and under similar irradiation reaction conditions 1,4bis[di(1H-indol-3-yl)methyl]benzene derivatives 5a-d were synthesized in good to excellent yields (Table 1) by the electrophilic substitution reaction of 2-arylindole derivatives 1a-d with terephthalaldehyde 4 (4:1 mmol) (Scheme 2).

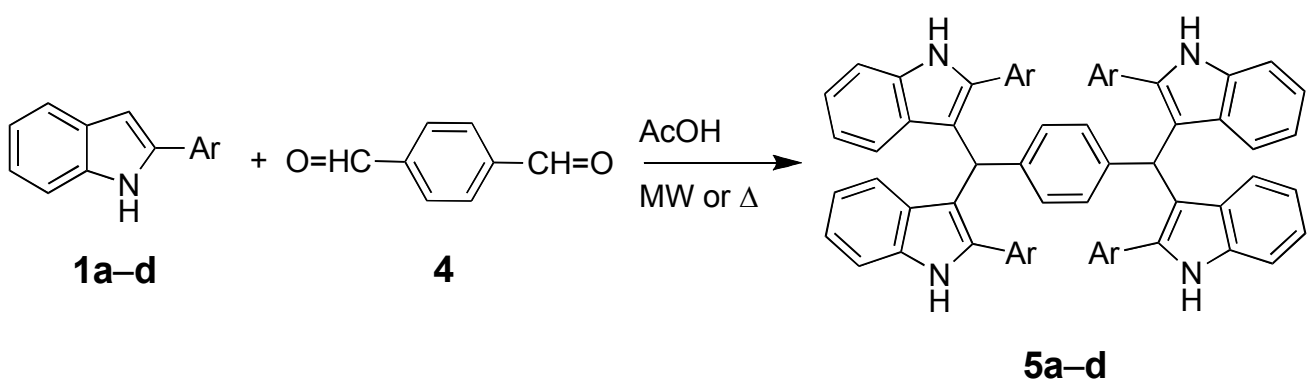

Scheme 2. Reaction of 2-arylindoles 1a-d with terephthaldehyde 4.

All products 3 and 5 were characterized by spectroscopic and elemental analyses. The IR spectra of di(1H-indol-3-yl)methanes 3 and 1,4-bis[di( $1 H$-indol-3-yl)methyl]benzene derivatives 5 show characteristic IR absorptions at 3747-3170 (N-H), 3062-3037 (aromatic C-H), 28602850 (aliphatic $\mathrm{C}-\mathrm{H}$ ), 1676-1660 (aromatic $\mathrm{C}=\mathrm{C}$ ), and 1613-1580 $(\mathrm{C}=\mathrm{C}-\mathrm{N}) \mathrm{cm}^{-1}$. In addition to substituent, aromatic and/or heteroaromatic proton signals, the ${ }^{1} \mathrm{H}$ NMR spectra display signals at $\delta$ 11.44-11.10 (br s, NH), and at $\delta 6.79-5.85(\mathrm{~s},>\mathrm{CH}-\mathrm{R}, \mathrm{R}=\operatorname{aryl}$, hetaryl) or 4.78-4.77 (t, $>\mathrm{CH}-\mathrm{R}, \mathrm{R}=n$-alkyl), respectively.

Melting points of di(1H-indol-3-yl)methanes $3 \mathrm{aa},{ }^{24,26} 3 \mathrm{ca},{ }^{25} 3 \mathrm{ac},{ }^{27} 3 \mathrm{bc},{ }^{27}$ and $3 \mathrm{cc}^{27}$ closely match those reported in the literature. No spectral data have been reported in the literature for compounds $3 \mathrm{ca}^{25} 3 \mathrm{bc},{ }^{27}$ and $3 \mathrm{cc}^{27}$; therefore, spectral data of these compounds are included in the Experimental Section.

All products 3 and 5 were obtained both by the microwave irradiation and conventional heating. Irrespective of these reaction conditions the IR spectra of each product are identical.

\section{Experimental Section}

General Procedures. All melting points were taken on a Stuart scientific melting point apparatus (Stuart Scientific, Stone, Staffordshire, UK). 1H NMR spectra of DMSO- $d_{6}$ solutions were recorded on a Varian Germini-2000 (300 MHz) spectrometer (Varian Inc., Palo Alto, CA, USA). IR spectra were recorded $(\mathrm{KBr})$ on a Pye-Unicam Sp-883 spectrophotometer, Microanalytical Laboratory, Faculty of Science, Cairo University. Elemental analyses $(\mathrm{C}, \mathrm{H}, \mathrm{N})$ were conducted using the Elemental Analyzer Yanaca CHN Corder MT-3. MS spectra were run on GC MS-QP 1000 EX Mass Spectrometer (Shimadzu). The microwave-induced reactions were carried out in an open Pyrex-glass vessel by using a domestic microwave oven (WhirlPool- 
TALENT). The synthesized products and each reaction carried out under conventionally or microwave (MW) irradiation condition were monitored by thinlayer chromatography (TLC) on Merck silica gel 60 F254 plates (type E; Merck) using UV light (254 and $360 \mathrm{~nm}$ ) for detection.

\section{Di(1H-indol-3-yl)methane derivatives 3. General procedures}

Microwave irradiation. A mixture of 2-arylindole $1 \mathbf{a}-\mathbf{d}$ (2 mmol), aldehyde $\mathbf{2 a}-\mathbf{g}(1 \mathrm{mmol})$ and glacial acetic acid $(1 \mathrm{~mL})$ in an open Pyrex-glass vessel was subjected to microwave irradiation (Table 1). Irradiation was carried out in successive $30 \mathrm{sec}$ periods to avoid overheating of the catalyst. After completion of the reaction as monitored by TLC, the reaction mixture was cooled, and poured onto water. The precipitated solid was filtered off, washed with water, dried and recrystallized.

Thermal conditions. A mixture of 2-arylindole $\mathbf{1 a}-\mathbf{d}(2 \mathrm{mmol})$, aldehyde $\mathbf{2 a}-\mathbf{g}(1 \mathrm{mmol})$ and glacial acetic acid $(1 \mathrm{~mL})$ was refluxed for the appropriate time (Table 1). After completion of the reaction as monitored by TLC, work-up was performed as described above.

3,3'-(Phenylmethylene)bis(2-phenyl-1H-indole) (3aa). Colorless crystals; mp 272-274 ${ }^{\circ} \mathrm{C}$ (methanol) (lit. ${ }^{24} 280{ }^{\circ} \mathrm{C}$; lit. $^{26} 261{ }^{\circ} \mathrm{C}$ ). IR (KBr): 3420, 3055, 2860, 1676, $1597 \mathrm{~cm}^{-1}$, ${ }^{1} \mathrm{H}$ NMR (300 MHz, DMSO- $d_{6}$ ): $\delta 11.31$ (br s, 2H, NH), 7.39-6.65 (m, 23H, ArH), 5.99 (s, 1H, CH). MS: $\mathrm{m} / \mathrm{z}(\%) 474(100)\left[\mathrm{M}^{+}\right]$. All the spectroscopic data match those reported. ${ }^{24,26}$

3,3'-(Phenylmethylene)bis(2-p-tolyl-1H-indole) (3ba). Colorless crystals; mp 250-252 ${ }^{\circ} \mathrm{C}$ (methanol). IR (KBr): 3439, 3052, 2860, 1660, $1613 \mathrm{~cm}^{-1} .{ }^{1} \mathrm{H}$ NMR (300 MHz, DMSO- $\left.d_{6}\right): \delta$ 11.25 (br s, 2H, NH), 7.39-6.6 (m, 21H, ArH), 6.01 (s, 1H, CH), 2.56 (s, 6H, CH $) . \mathrm{MS}: \mathrm{m} / \mathrm{z}(\%)$ 502 (2.1) $\left[\mathrm{M}^{+}\right]$. Anal. calcd. for $\mathrm{C}_{37} \mathrm{H}_{30} \mathrm{~N}_{2}$ : C, 88.41; H, 6.02; N, 5.57. Found: C, 88.48; H, 6.28; N, 5.46 .

3,3'-(Phenylmethylene)bis[2-(4-fluorophenyl)-1H-indole] (3ca). Colorless crystals; mp 220$221{ }^{\circ} \mathrm{C}\left(\mathrm{CHCl}_{3} /\right.$ petroleum ether) (lit. $\left.{ }^{25} 215{ }^{\circ} \mathrm{C}\right)$. IR (KBr): 3745, 3057, 2364, 1657, $1604 \mathrm{~cm}^{-1}$. ${ }^{1} \mathrm{H}$ NMR (300 MHz, DMSO-d $)_{6}$ : $\delta 11.29$ (s, 2H, NH), 7.36-6.67 (m, 21H, ArH), 5.89 (s, 1H, $\mathrm{CH})$. MS: $\mathrm{m} / \mathrm{z}(\%) 510(100)\left[\mathrm{M}^{+}\right]$. Anal. calcd. for $\mathrm{C}_{35} \mathrm{H}_{24} \mathrm{~F}_{2} \mathrm{~N}_{2}$ : C, 82.33; H, 4.74; N, 5.49. Found: C, 82.48; H, 4.97; N, 5.22.

3,3'-(Phenylmethylene)bis[2-(4-chlorophenyl)-1H-indole] (3da). Colorless crystals; mp 270$272{ }^{\circ} \mathrm{C}\left(\mathrm{CHCl}_{3}\right)$. IR (KBr): 3425, 3058, 1903, 1675, $1600 \mathrm{~cm}^{-1} .{ }^{1} \mathrm{H}$ NMR (300 MHz, DMSO- $\left.d_{6}\right)$ : $\delta 11.33(\mathrm{~s}, 2 \mathrm{H}, \mathrm{NH}), 7.36-6.68(\mathrm{~m}, 21 \mathrm{H}, \mathrm{ArH}), 5.95$ (s, 1H, CH). MS: m/z (\%) $542(48.3)\left[\mathrm{M}^{+}\right]$. Anal. calcd. for $\mathrm{C}_{35} \mathrm{H}_{24} \mathrm{Cl}_{2} \mathrm{~N}_{2}$ : C, 77.35; H, 4.45; N, 5.15. Found: C, 77.22; H, 4.65; N, 5.21.

3,3'-(3-Nitrophenylmethylene)bis(2-phenyl-1H-indole) (3ab). Yellow crystals; mp 269-271 ${ }^{\circ} \mathrm{C}$ (methanol). IR (KBr): 3444, 3056, 2860, 1660, $1605 \mathrm{~cm}^{-1} .{ }^{1} \mathrm{H}$ NMR (300 MHz, DMSO- $\left.d_{6}\right): \delta$ 11.43 (br s, 2H, NH), 8.10-6.69 (m, 22H, ArH), 6.12 (s, 1H, CH). MS: m/z (\%) $519(100)\left[\mathrm{M}^{+}\right]$. Anal. calcd. for $\mathrm{C}_{35} \mathrm{H}_{25} \mathrm{~N}_{3} \mathrm{O}_{2}:$ C, 80.91; H, 4.85; N, 8.09. Found: C, 80.34; H, 5.11; N, 7.98.

3,3'-(3-Nitrophenylmethylene)bis(2-p-tolyl-1H-indole) (3bb). Yellow crystals; mp 247-249 ${ }^{\circ} \mathrm{C}$ (methanol). IR (KBr): 3246 2921, 2860, 1659, $1613 \mathrm{~cm}^{-1} .{ }^{1} \mathrm{H}$ NMR (300 MHz, DMSO- $\left.d_{6}\right): \delta$ 11.35 (br s, 2H, NH), 8.10-6.68 (m, 20H, ArH), 6.11 (s, 1H, CH), 2.25 (s, 6H, $\left.\mathrm{CH}_{3}\right) . \mathrm{MS}: \mathrm{m} / \mathrm{z}$ 
(\%) 547 (100) $\left[\mathrm{M}^{+}\right]$. Anal. calcd. for $\mathrm{C}_{37} \mathrm{H}_{29} \mathrm{~N}_{3} \mathrm{O}_{2}$ : C, 81.15; H, 5.34; N, 7.67. Found: C, 81.25; H, 5.60; N, 7.56.

3,3'-(3-Nitrophenylmethylene)bis[2-(4-fluorophenyl)-1H-indole] (3cb). Yellow crystals; mp 250-252 ${ }^{\circ} \mathrm{C}$ (ethanol). IR (KBr): 3746, 3060, 2363, 1647, $1613 \mathrm{~cm}^{-1}$. ${ }^{1} \mathrm{H}$ NMR (300 MHz, DMSO-d $\left.d_{6}\right): \delta 11.40(\mathrm{~s}, 2 \mathrm{H}, \mathrm{NH}), 7.93-6.71(\mathrm{~m}, 20 \mathrm{H}, \mathrm{ArH}), 6.04(\mathrm{~s}, 1 \mathrm{H}, \mathrm{CH}) . \mathrm{MS}: \mathrm{m} / \mathrm{z}(\%) 555$ (100) $\left[\mathrm{M}^{+}\right]$. Anal. calcd. for $\mathrm{C}_{35} \mathrm{H}_{23} \mathrm{~F}_{2} \mathrm{~N}_{3} \mathrm{O}_{2}$ : C, 75.67; H, 4.17; N, 7.56. Found: C, 75.82; H, 4.30; N, 7.29.

3,3'-(3-Nitrophenylmethylene)bis[2-(4-chlorophenyl)-1H-indole] (3db). Yellow crystals; mp $>300{ }^{\circ} \mathrm{C}$ (methanol/DMF). IR (KBr): 3745, 3059, 2363, 1647, $1527 \mathrm{~cm}^{-1} .{ }^{1} \mathrm{H}$ NMR (300 MHz, DMSO-d $\left.)_{6}\right): \delta 11.44$ (s, 2H, NH), 7.97-6.72 (m, 20H, ArH), 6.10 (s, 1H, CH). MS: m/z (\%) 587 (42.8) $\left[\mathrm{M}^{+}\right]$. Anal. calcd. for $\mathrm{C}_{35} \mathrm{H}_{23} \mathrm{Cl}_{2} \mathrm{~N}_{3} \mathrm{O}_{2}$ : C, 71.43; H, 3.94; N, 7.14. Found: C, 71.53; H, $4.07 ; \mathrm{N}, 6.87$.

3,3'-(2-Furylmethylene)bis(2-phenyl-1H-indole) (3ac). Colorless crystals; mp 256-257 ${ }^{\circ} \mathrm{C}$ (methanol) (Lit. ${ }^{27} 255^{\circ} \mathrm{C}$ ). Spectroscopic data (IR, ${ }^{1} \mathrm{H}$ NMR, and MS) match those reported. ${ }^{27}$

3,3'-(2-Furylmethylene)bis(2-p-tolyl-1H-indole) (3bc). Colorless crystals; $\mathrm{mp}>300{ }^{\circ} \mathrm{C}$ (methanol) $\left(\right.$ Lit. $^{27}>360{ }^{\circ} \mathrm{C}$ ). IR (KBr): 3422, 2861, 1915, 1669, $1449 \mathrm{~cm}^{-1} .{ }^{1} \mathrm{H}$ NMR (300 MHz, DMSO-d $\left.)_{6}\right): \delta 11.21$ (br s, 2H, NH), 7.95-5.92 (m, 19H, ArH), $5.84(\mathrm{~s}, 1 \mathrm{H}, \mathrm{CH}), 2.27$ (s, 6H, $\left.\mathrm{CH}_{3}\right)$. MS: m/z (\%) $492(100)\left[\mathrm{M}^{+}\right]$. Anal. calcd. for $\mathrm{C}_{35} \mathrm{H}_{28} \mathrm{~N}_{2} \mathrm{O}: \mathrm{C}, 85.34 ; \mathrm{H}, 5.73 ; \mathrm{N}, 5.69$. Found: C, 85.41; H, 5.99; N, 5.58.

3,3'-(2-Furylmethylene)bis [2-(4-fluorophenyl)-1 $\boldsymbol{H}$-indole] (3cc). Colorless crystals; mp >300 ${ }^{\circ} \mathrm{C}\left(\mathrm{CHCl}_{3}\right)\left(\right.$ Lit. $\left.^{27}>360{ }^{\circ} \mathrm{C}\right)$. IR (KBr): 3745, 3056, 2362, 1647, $1603 \mathrm{~cm}^{-1}$. ${ }^{1} \mathrm{H} \mathrm{NMR}(300 \mathrm{MHz}$, DMSO-d $\left.)_{6}\right): \delta 11.29$ (s, 2H, NH), 7.55-5.96 (m, 19H, ArH), 5.78 (s, 1H, CH). MS: m/z (\%) 500 (100) $\left[\mathrm{M}^{+}\right]$. Anal. calcd. for $\mathrm{C}_{33} \mathrm{H}_{22} \mathrm{~F}_{2} \mathrm{~N}_{2} \mathrm{O}: \mathrm{C}, 79.19 ; \mathrm{H}, 4.43 ; \mathrm{N}, 5.60$. Found: C, 79.34; H, 4.26; $\mathrm{N}, 5.33$.

3,3'-(2-Furylmethylene)bis [2-(4-chlorophenyl)-1H-indole] (3dc). Colorless crystals; mp 256$258{ }^{\circ} \mathrm{C}\left(\mathrm{CHCl}_{3}\right)$. IR (KBr): 3745, 3054, 1892, 1648, $1598 \mathrm{~cm}^{-1} .{ }^{1} \mathrm{H}$ NMR (300 MHz, DMSO- $\left.d_{6}\right)$ :

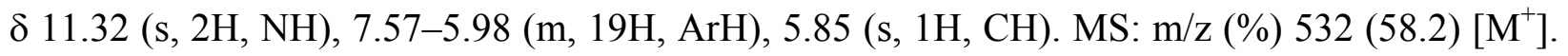
Anal. calcd. for $\mathrm{C}_{33} \mathrm{H}_{22} \mathrm{Cl}_{2} \mathrm{~N}_{2} \mathrm{O}$ : C, 74.30; H, 4.16; N, 5.25. Found: C, 74.45; H, 4.39; N, 4.98 .

3,3'-(3-Pyridylmethylene)bis(2-phenyl-1H-indole) (3ad). Colorless crystals; $\mathrm{mp}>300{ }^{\circ} \mathrm{C}$ (methanol). IR (KBr): 3387, 3161, 1890, 1663, $1578 \mathrm{~cm}^{-1} .{ }^{1} \mathrm{H}$ NMR (300 MHz, DMSO- $\left.d_{6}\right): \delta$ 11.40 (br s, 2H, NH), 8.44-6.69 (m, 22H, ArH), 6.06 (s, 1H, CH). MS: m/z (\%) 475 (92.6) [M $\left.{ }^{+}\right]$. Anal. calcd. for $\mathrm{C}_{34} \mathrm{H}_{25} \mathrm{~N}_{3}$ : C, 85.87; H, 5.30; N, 8.84. Found: C, 85.94; H, 5.58; N, 8.73.

3,3'-(3-Pyridylmethylene)bis(2-p-tolyl-1H-indole) (3bd). Colorless crystals; mp 299-300 ${ }^{\circ} \mathrm{C}$ (methanol). IR (KBr): 3170, 2924, 2850, 1661, $1580 \mathrm{~cm}^{-1} .{ }^{1} \mathrm{H}$ NMR (300 MHz, DMSO- $\left.d_{6}\right): \delta$ 11.30 (br s, 2H, NH), 8.43-6.66 (m, 20H, ArH), 6.03 (s, 1H, CH), $2.26\left(\mathrm{~s}, 6 \mathrm{H}, \mathrm{CH}_{3}\right) . \mathrm{MS}: \mathrm{m} / \mathrm{z}$ (\%) 503 (100) $\left[\mathrm{M}^{+}\right]$. Anal. calcd. $\mathrm{C}_{36} \mathrm{H}_{29} \mathrm{~N}_{3}$ : C, 85.85; H, 5.80; N, 8.34. Found: C, 85.92; H, 6.06; N, 8.23.

3,3'-(3-Pyridylmethylene)bis[2-(4-fluorophenyl)-1H-indole] (3cd). Colorless crystals; mp $>300{ }^{\circ} \mathrm{C}$ (methanol/DMF). IR (KBr): 3745, 2835, 1693, 1647, $1615 \mathrm{~cm}^{-1}$. ${ }^{1} \mathrm{H}$ NMR (300 MHz, DMSO-d $\left.d_{6}\right): \delta 11.36(\mathrm{~s}, 2 \mathrm{H}, \mathrm{NH}), 8.44-6.68(\mathrm{~m}, 20 \mathrm{H}, \mathrm{ArH}), 5.96(\mathrm{~s}, 1 \mathrm{H}, \mathrm{CH}) . \mathrm{MS}: \mathrm{m} / \mathrm{z}(\%) 511$ 
(100) $\left[\mathrm{M}^{+}\right]$. Anal. calcd. for $\mathrm{C}_{34} \mathrm{H}_{23} \mathrm{~F}_{2} \mathrm{~N}_{3}: \mathrm{C}, 79.83 ; \mathrm{H}, 4.53 ; \mathrm{N}, 8.21$. Found: $\mathrm{C}, 79.98 ; \mathrm{H}, 4.36 ; \mathrm{N}$, 7.94 .

3,3'-(3-Pyridylmethylene)bis[2-(4-chlorophenyl)-1H-indole] (3dd). Colorless crystals; mp $>300{ }^{\circ} \mathrm{C}$ (methanol/DMF). IR (KBr): 3401, 3049, 1900, 1667, $1576 \mathrm{~cm}^{-1} .{ }^{1} \mathrm{H}$ NMR $(300 \mathrm{MHz}$, DMSO- $\left.d_{6}\right): \delta 11.39$ (br s, $\left.2 \mathrm{H}, \mathrm{NH}\right), 8.38-6.72(\mathrm{~m}, 20 \mathrm{H}, \mathrm{ArH}), 6.03$ (s, 1H, CH). MS: m/z (\%) $543(27.1)\left[\mathrm{M}^{+}\right]$. Anal. calcd. for $\mathrm{C}_{34} \mathrm{H}_{23} \mathrm{Cl}_{2} \mathrm{~N}_{3}: \mathrm{C}, 75.00 ; \mathrm{H}, 4.26 ; \mathrm{N}, 7.72$. Found: $\mathrm{C}, 75.15 ; \mathrm{H}$, $4.09 ; \mathrm{N}, 7.45$.

3,3'-(1-Pyrenylmethylene)bis(2-phenyl-1H-indole) (3ae). Colorless crystals; $\mathrm{mp}>300{ }^{\circ} \mathrm{C}$ (methanol). IR (KBr): 3288, 3049, 1676, 1640, $1597 \mathrm{~cm}^{-1} .{ }^{1} \mathrm{H}$ NMR (300 MHz, DMSO- $d_{6}$ ): $\delta$ 11.38 (br s, 2H, NH), 8.28-6.51 (m, 27H, ArH), 6.26 (br s, 1H, CH). MS: m/z (\%) 598 (42.9) $\left[\mathrm{M}^{+}\right]$. Anal. calcd. for $\mathrm{C}_{45} \mathrm{H}_{30} \mathrm{~N}_{2}: \mathrm{C}, 90.27 ; \mathrm{H}, 5.05 ; \mathrm{N}, 4.68$. Found: $\mathrm{C}, 90.34 ; \mathrm{H}, 5.31 ; \mathrm{N}, 4.57$.

3,3'-(1-Pyrenylmethylene)bis(2-p-tolyl-1H-indole) (3be). Colorless crystals; mp $268-270{ }^{\circ} \mathrm{C}$ (methanol). IR (KBr): 3413, 3040, 1676, 1649, $1597 \mathrm{~cm}^{-1} .{ }^{1} \mathrm{H}$ NMR (300 MHz, DMSO- $\left.d_{6}\right): \delta$ 11.27 (br s, 2H, NH), 8.26-6.94 (m, 25H, ArH), 6.79 (s, 1H, CH), 2.19 (s, 6H, $\mathrm{CH}_{3}$ ). MS: m/z (\%) $626(43.4)\left[\mathrm{M}^{+}\right]$. Anal. calcd. for $\mathrm{C}_{47} \mathrm{H}_{34} \mathrm{~N}_{2}$ : C, 90.06; H, 5.47; N, 4.47. Found: C, 90.13; H, $5.73 ; \mathrm{N}, 4.36$.

3,3'-(1-Pyrenylmethylene)bis[2-(4-fluorophenyl)-1H-indole] (3ce). Pale yellow crystals; mp 248-250 ${ }^{\circ} \mathrm{C}$ (methanol). IR (KBr): 3745, 3047, 2362, 1693, $1647 \mathrm{~cm}^{-1} .{ }^{1} \mathrm{H}$ NMR $(300 \mathrm{MHz}$, DMSO- $\left.d_{6}\right): \delta 11.43$ (br s, 2H, NH), 8.27-7.00 (m, 25H, ArH), 6.70 (br s, $\left.1 \mathrm{H}, \mathrm{CH}\right) . \mathrm{MS}: \mathrm{m} / \mathrm{z}(\%)$ $634(32.2)\left[\mathrm{M}^{+}\right]$. Anal. calcd. for $\mathrm{C}_{45} \mathrm{H}_{28} \mathrm{~F}_{2} \mathrm{~N}_{2}: \mathrm{C}, 85.15 ; \mathrm{H}, 4.45 ; \mathrm{N}, 4.41$. Found: $\mathrm{C}, 85.02 ; \mathrm{H}$, 4.65; N, 4.47 .

3,3'-(1-Pyrenylmethylene)bis[2-(4-chlorophenyl)-1H-indole] (3de). Pale yellow crystals; mp 250-252 ${ }^{\circ} \mathrm{C}$ (methanol). IR (KBr): 3746, 3037, 2856, 1657, $1483 \mathrm{~cm}^{-1} .{ }^{1} \mathrm{H}$ NMR $(300 \mathrm{MHz}$, DMSO- $d_{6}$ ): $\delta 11.44$ (br s, 2H, NH), 8.26-7.20 (m, 25H, ArH), 6.73 (br s, $\left.1 \mathrm{H}, \mathrm{CH}\right), 2.26(\mathrm{~s}, 6 \mathrm{H}$, $\left.\mathrm{CH}_{3}\right)$. MS: $\mathrm{m} / \mathrm{z}(\%) 668(25.2)\left[\mathrm{M}^{+}\right]$. Anal. calcd. for $\mathrm{C}_{45} \mathrm{H}_{28} \mathrm{Cl}_{2} \mathrm{~N}_{2}: \mathrm{C}, 80.69 ; \mathrm{H}, 4.23 ; \mathrm{N}, 4.20$. Found: C, 80.51; H, 4.43; N, 4.26.

3,3'-(1-Heptylmethylene)bis(2-phenyl-1H-indole) (3af). Colorless crystals; mp $134-136{ }^{\circ} \mathrm{C}$ (ethanol). IR (KBr): 3747, 3056, 2856, 1657, $1604 \mathrm{~cm}^{-1} .{ }^{1} \mathrm{H}$ NMR (300 MHz, DMSO- $\left.d_{6}\right): \delta$ 11.10 (s, 2H, NH), 7.54-6.78 (m, 18H, ArH), 4.78 (t, 1H, CH), 2.27 (q, 2H, $\left.\mathrm{CH}_{2}\right), 1.08-1.00$ (m, $\left.8 \mathrm{H}, \mathrm{CH}_{2}\right), 0.71$ (t, 3H, $\left.\mathrm{CH}_{3}\right)$. MS: $\mathrm{m} / \mathrm{z}(\%) 482(18.04)\left[\mathrm{M}^{+}\right]$. Anal. calcd. for $\mathrm{C}_{35} \mathrm{H}_{34} \mathrm{~N}_{2}: \mathrm{C}, 87.10$; H, 7.10; N, 5.80. Found: C, 87.17; H, 7.36; N, 5.82.

3,3'-(1-Nonylmethylene)bis(2-phenyl-1H-indole) (3ag). Colorless crystals; mp $138-140{ }^{\circ} \mathrm{C}$ (ethanol). IR (KBr): 3400, 3055, 2923, 2852, $1605 \mathrm{~cm}^{-1} .{ }^{1} \mathrm{H}$ NMR (300 MHz, DMSO- $\left.d_{6}\right): \delta$ 11.10 (s, 2H, NH), 7.54-6.77 (m, 18H, ArH), 4.77 (t, 1H, CH), 2.27 (q, 2H, $\left.\mathrm{CH}_{2}\right), 1.17-1.03$ (m, $\left.12 \mathrm{H}, \mathrm{CH}_{2}\right), 0.79\left(\mathrm{t}, 3 \mathrm{H}, \mathrm{CH}_{3}\right)$. MS: $\mathrm{m} / \mathrm{z}(\%) 510(9.7)\left[\mathrm{M}^{+}\right]$. Anal. calcd. for $\mathrm{C}_{37} \mathrm{H}_{38} \mathrm{~N}_{2}: \mathrm{C}, 87.02$; H, 7.50; N, 5.48. Found: C, 87.09; H, 7.76; N, 5.37.

3,3'-(1-Heptylmethylene)bis [2-(4-chlorophenyl)-1H-indole] (3df). Colorless crystals; mp 196$198^{\circ} \mathrm{C}$ (ethanol). IR (KBr): 3744, 2953, 2855, 1693, $1647 \mathrm{~cm}^{-1}$. ${ }^{1} \mathrm{H}$ NMR (300 MHz, DMSO- $\left.d_{6}\right)$ : $\delta 11.09(\mathrm{~s}, 2 \mathrm{H}, \mathrm{NH}), 7.65-6.82(\mathrm{~m}, 16 \mathrm{H}, \mathrm{ArH}), 4.72(\mathrm{t}, 1 \mathrm{H}, \mathrm{CH}), 2.38\left(\mathrm{q}, 2 \mathrm{H}, \mathrm{CH}_{2}\right), 1.21-1.05$ 
$\left(\mathrm{m}, 8 \mathrm{H}, \mathrm{CH}_{2}\right), 0.78\left(\mathrm{t}, 3 \mathrm{H}, \mathrm{CH}_{3}\right) . \mathrm{MS}: \mathrm{m} / \mathrm{z}(\%) 550(9.7)\left[\mathrm{M}^{+}\right]$. Anal. calcd. for $\mathrm{C}_{35} \mathrm{H}_{32} \mathrm{Cl}_{2} \mathrm{~N}_{2}$ : C, 76.22; H, 5.85; N, 5.08. Found: C, 76.09; H, 6.05; N, 5.14.

3,3'-(1-Nonylmethylene)bis[2-(4-chlorophenyl)-1H-indole] (3dg). Colorless crystals; mp 150$152^{\circ} \mathrm{C}$ (ethanol). IR (KBr): 3744, 2923, 2852, 1693, $1647 \mathrm{~cm}^{-1} .{ }^{1} \mathrm{H}$ NMR (300 MHz, DMSO- $\left.d_{6}\right)$ : $\delta 11.09$ (s, 2H, NH), 7.64-6.84 (m, 16H, ArH), 4.72 (t, 1H, CH), 2.35 (q, 2H, $\mathrm{CH}_{2}$ ), 1.19-1.09 $\left(\mathrm{m}, 8 \mathrm{H}, \mathrm{CH}_{2}\right), 0.83\left(\mathrm{t}, 3 \mathrm{H}, \mathrm{CH}_{3}\right) . \mathrm{MS}: \mathrm{m} / \mathrm{z}(\%) 578(9.4)\left[\mathrm{M}^{+}\right]$. Anal. calcd. for $\mathrm{C}_{35} \mathrm{H}_{32} \mathrm{Cl}_{2} \mathrm{~N}_{2}$ : $\mathrm{C}$, 76.67; H, 6.26; N, 4.83. Found: C, 76.54; H, 6.46; N, 4.89 .

\section{1,4-Bis[bis(2-aryl-1H-indol-3-yl)methyl]benzene derivatives 5. General procedures}

Microwave irradiation. A mixture of 2-arylindole 1a-d $(4 \mathrm{mmol})$, terephthalaldehyde 4 (1 $\mathrm{mmol})$ and glacial acetic acid $(1 \mathrm{ml})$ was subjected to the same reaction conditions as described above for the preparation of $\mathbf{3}$.

Thermal conditions. A mixture of 2-arylindole 1a-d (4 mmol), terephthalaldehyde 4 ( $1 \mathrm{mmol})$ and glacial acetic acid $(1 \mathrm{ml})$ was subjected to the same reaction conditions as described above for the preparation of $\mathbf{3}$.

1,4-Bis[bis(2-phenyl-1H-indol-3-yl)methyl]benzene (5a). Colorless crystals; $\mathrm{mp}>300{ }^{\circ} \mathrm{C}$ (methanol). IR (KBr): 3297, 3060, 2860, 1661, $1602 \mathrm{~cm}^{-1} .{ }^{1} \mathrm{H}$ NMR (300 MHz, DMSO- $\left.d_{6}\right): \delta$ 11.31 (br s, 4H, NH), 7.95-6.67 (m, 40H, ArH), 5.92 (s, 2H, CH). MS: m/z (\%) 870 (5.9) [M ${ }^{+}$]. Anal. calcd. for $\mathrm{C}_{64} \mathrm{H}_{46} \mathrm{~N}_{4}$ : C, 88.25; H, 5.32; N, 6.43. Found: C, 88.32; H, 5.58; N, 6.32.

1,4-Bis[bis[2-(4-methylphenyl)-1H-indol-3-yl]methyl]benzene (5b). Colorless crystals; mp $>300{ }^{\circ} \mathrm{C}$ (methanol). IR (KBr): 3430, 3051, 2913, 1661, $1549 \mathrm{~cm}^{-1} .{ }^{1} \mathrm{H}$ NMR (300 MHz, DMSO$\left.d_{6}\right): \delta 11.21$ (br s, 4H, NH), 7.35-6.66 (m, 36H, ArH), 5.88 (s, 2H, CH), $2.27\left(\mathrm{~s}, 12 \mathrm{H}, \mathrm{CH}_{3}\right)$. MS: $\mathrm{m} / \mathrm{z}(\%) 927$ (33.3) $\left[\mathrm{M}^{+}\right]$. Anal. calcd. for $\mathrm{C}_{68} \mathrm{H}_{54} \mathrm{~N}_{4}$ : C, 88.09; H, 5.87; N, 6.04. Found: C, 88.16; H, 6.13; N, 5.93.

1,4-Bis[bis[2-(4-fluorophenyl)-1H-indol-3-yl]methyl]benzene (5c). Colorless crystals; mp $>300{ }^{\circ} \mathrm{C}$ (methanol/DMF). IR (KBr): 3746, 3056, 2912, 1694, $1647 \mathrm{~cm}^{-1} .{ }^{1} \mathrm{H}$ NMR (300 MHz, DMSO-d d $: \delta 11.39$ (br s, 4H, NH), 8.38-6.72 (m, 36H, ArH), 6.03 (s, 2H, CH). MS: m/z (\%) 942 (60) $\left[\mathrm{M}^{+}\right]$. Anal. calcd. for $\mathrm{C}_{64} \mathrm{H}_{42} \mathrm{~F}_{4} \mathrm{~N}_{4}$ : C, 81.51; H, 4.49; N, 5.94. Found: C, 81.38; H, 4.69; $\mathrm{N}, 6.00$.

1,4-Bis[bis[2-(4-chlorophenyl)-1H-indol-3-yl]methyl]benzene (5d). Colorless crystals; mp $>300{ }^{\circ} \mathrm{C}$ (methanol/DMF). IR (KBr): 3746, 3380, 2363, 1676, $1648 \mathrm{~cm}^{-1} .{ }^{1} \mathrm{H}$ NMR (300 MHz, DMSO-d $\left.)_{6}\right): \delta 11.34$ (br s, 4H, NH), 7.95-6.67 (m, 36H, ArH), 5.91 (s, 2H, CH). MS: m/z (\%) 1006 (33) $\left[\mathrm{M}^{+}\right]$. Anal. calcd. for $\mathrm{C}_{64} \mathrm{H}_{42} \mathrm{Cl}_{4} \mathrm{~N}_{4}$ : C, 76.19; H, 4.20; N, 5.55. Found: C, 76.06; H, $4.40 ; \mathrm{N}, 5.61$. 


\section{References and Notes}

1. Sundberg, R. J. The Chemistry of Indoles; Academic Press: New York, 1996; p 113.

2. (a) Kathleen, A.; Merrill, A. G. PCT. Int. Appl. WO 99; Chem. Abstr. 1999, 130, 276765.

(b) Bradfield, C. A.; Bjeldanes, L. F. J. Toxicol. Environ. Health 1987, 21, 311. (c) Dashwood, R. H.; Uyetake, L.; Fong, A. T.; Hendricks, J. D.; Bailey, G. S. Food Chem. Toxicol. 1987, 27, 385.

3. (a) Xiaokang, Ge; Fares, F. A.; Yannai, S. Anticancer Research 1999, 19, 3199. (b) Chang, Yu-Chen; Riby, J.; Chang, Grace H. F.; Peng, G. F.; Bjeldanes, L. F. Biochem. Pharmacol. 1999, 58, 825.

4. (a) Shilling, A. D.; Carlson, D. B.; Katchamart, S.; Williams, D. Appl.Pharmacol. 2001, 170, 191. (b) Ge, X.; Yannai, S.; Rennert, G.; Gruener, N.; Fares, F. A. Biochem. Biophys. Res. Commun. 1996, 228, 153. (c) Ge, X.; Fares, F. A.; Yannai, S. Anticancer Res. 1999, 19, 3199.

5. Remers, W. A.; Houlihan, W. J., Eds.; Heterocyclic Compounds, Interscience Publishers: New York, 1972; p 1.

6. (a) Noland, W. E.; Venkiteswaran, M. R.; Richards, C. G.; J. Org. Chem. 1961, 26, 4241.

(b) Banerji, J.; Chatterjiee, A.; Manna, S.; Pascard, C.; Prange, T.; Shoolery. J. Heterocycles $1981,15,325$.

7. Chatterjee, A.; Manna, S.; Benerji, T.; Shoolery, J. J. Chem. Soc., Perkin I 1980, 553.

8. Chen, D.; Yu, L.; Wang, P. G. Tetrahedron Lett. 1996, 37, 4467.

9. Babu, G.; Sridhar, N.; Petrumal, P. T. Synth.Commun. 2000, 30, 1609.

10. Koshima, H.; Matsuaka, W. J. Heterocyclic. Chem. 2002, 39, 1089.

11. Reddy, A. V.; Ravinder, K.; Reddy, V. L. N.; Goud, T. V.; Ravikant, V.; Venkateswarlu, Y. Synth. Commun. 2003, 33, 3687.

12. Wang, L.; Han, J.; Tian, H.; Sheng, J.; Fan, Z.; Tang, X. Synlett 2005, 337.

13. Mi, X.; Luo, S.; He, J.; Cheng, J.-P. Tetrahedron Lett. 2004, 45, 4567.

14. Ji, S.-J.; Zhou, M.- F.; Gu, D.-G.; Jiang, Z.-Q.; Loh, T.-P. Eur. J. Chem. 2004, 1584.

15. Yadav, J.S.; Reddy, B.S.; Sunita, S. Adv. Synth. Catal. 2003, 3, 349.

16. Hermken, S. P.; Ottenheijm, H.; Ress, D. Tetrahedron 1997, 53, 5643.

17. (a) Jeselnik, M.; Varma, R. S.; Polanc, S.; Kocevar, M. Green Chem. 2002, 4, 35. (b) Lidstrom, P.; Tierney, J.; Wathey, B.; Westman, J. Tetrahedron 2001, 57, 9225. (c) Loupy, A.; Perreux, L. Tetrahedron 2001, 57, 9199. (d) Hoz, A. De L. A.; D.-Ortis, A.; Moreno, A.; Langa, F. M. Eur. J. Org. Chem. 2000, 3659. (e) Elander, N.; Jones, J. R.; Lu, S.-Y.; StoneElender, S. Chem. Soc. Rev. 2000, 29, 239. (f) Deshayes, S.; Liagre, M.; Loupy, A.; Luche, J.; Petit, A. Tetrahedron 1999, 55, 10851. (g) Varma, R. S. Green Chem. 1999, 1, 43.

18. Dallinger, D.; Kappe, C. O. Chem. Rev. 2007, 107, 2563.

19. (a) Zahran, M. A.-H.; Afify, H. M.; Pedersen, E. B.; Nielsen, C. J. Chem. Research (M) 2001, 0101. (b) Zahran, M. A.-H.; El-Sawy, E. R.; Ebid, M. S.; El-Tablawy, S. Y. Egypt. Pharm. J. (NRC). 2007, 6, 13. 
20. Zahran, M. A.-H.; El-Essawy, F. A.-A.; Yassin, S. M.; Salem, T. A.-R.; Boshta. N. M. Arch. Pharm. Chem. Life Sci. 2007, 340, 591.

21. Shun-Jun, J.; Shun-Yi, W.; Yong, Z.; Tech-Peng, L. Tetrahedron 2004, 60, 2051.

22. Pore, D. M.; Desai, U. V.; Thopate, T. S.; Watgaonkar, P. P. ARKIVOC 2006, (xii), 75.

23. Kamble, V. T.; Kadam, K. R.; Joshi, N. S.; Muley, D. B. Catal. Commun. 2007, 8, 498.

24. Berti, C; Greci, L; Marchetti, L. J. Heterocycl. Chem. 1978, 15, 433.

25. Joshi, K. C.; Pathak, V. N.; Chand, P. Indian. J. Chem. 1978, 16B, 933.

26. Banerji, P.; Mukhopadhyay, A. K. Indian. J. Chem. 1982, 21B, 293.

27. Pathak, V. N.; Gupta, R.; Garg, M.; Rao, V. M. Indian. J. Heterocycl. Chem. 2001, 11, 107. 